\title{
Tumor de Wilms: Análisis de 23 casos
}

\author{
Dres. Galicia Montecinos Latorre*; Mireya Bravo**; \\ Gustavo Aldunate Nốel*; David Mirkin W.**; \\ Carlos Vild6sola****
}

\section{MATERIAL Y METODO}

La incirlencia de tumores malignos cono cauincremento progresivo en todo el mundo, en forma relativá y absoluta (5). Los niños también siguen esta tendencia, especialmente por tumores de tipo embrionario (3).

Las estadísticas extranjeras relativas a las lesiones malignas de la infancia, muestran que los tumores de Wilms representan un 20 a $30 \%$ de todos ellos $(9,19)$ y un $6 \%$ de todos los tumores renales a cualquier edad (5).

Las estadísticas parciales chilenas le asignan al tumor de Wilms un $10 \%$ te la totalirlad de Ias neoplasias malignas infantiles (46-37) y un $10 \%$ de los tumores renales (4). Su elevada mortalidad $(42 \times 100.000)$ en la población menor de 15 años $(34,99)$ le da un pronóstico sombrio. Esto ha motivado la investigación de nuevas modalidades terapéuticas $(27,34,37,39)$ tendientes a lograr una mayor sobrevida.

En el presente trabajo revisamos nuestra casuística de los últimos años con el objeto de destacar los hullazgos clínicos y analizar el tratamicnio efectuado para evaluar sus resultados a la luz de nuevas pautas terapéuticas propuestas por diferentes autores en los últimos años. Rio.

* Servicio de Cirugia Infantil. Hospital Roberto del

* Departamento de Pediatría, Hospital R, del Rio. * *Servicio Anatomía Patológíca, Hospital R. deI Rto.

***Servicio de Radiologfa, Hospital R. del Rio.

Rev. Chilena de Pediatría, Vol. 47, NP 1, 1976
Se analizan las fichas clínicas de 23 enfermos que ingresan al Hospital Roberto del Río entre los años 1962-1974 y en los que se comprobó un tumor de Wilms.

El estudio de estos pacientes se basó cn el examen clínico, complementado con radiografía de abdomen simple y pielografía de eliminación, además de algunos otros exámenes como hemograma, VSH, orina completa etc.

Todos los enfermos fueron intervenidos quirúrgicamente, recibiendo la mayoria de ellos quimioterapia y radioterapia según la modalidad clínica de la lesión, los resultados operatorios y anatomopatológicos y según la disponibilidad de drogas.

El estudio histopatológico se efectuó en tockos los casos y su resultado determinó el tratamiento postoperatorio a seguir.

\section{RESULTADOS}

La distribución por sexo y por edad coincide con cifras encontradas en diferentes publica. ciones $(1,3,9,11)$, el tumor se presenta con mayor frecuencia er. los menores de dos años sin diferencia en cuanto a sexo.

El análisis de los sín tomas muestra habitualmente un desarrollo insidioso e indoloro de la lesión hasta que el tumor es evidente y se cletecta como masa palpable retroperitoneal. En el $60 \%$ de los casos los síntomas comenzaron entre 2-3 meses antes del ingreso y el motivo de consulta más frecuente fue un au- 
mento de volumen abdominal en fosa lumbar y/o flanco.

En 5 casos cste aumento de volumen fue hallazgo durante un examen físico motivado por otra patología. Lá hematuria y el color abdominal se presentaron con menor frecuencia, como asimismo el compromiso del estado general. Solamente en un caso se detectó una hipertensión arterial.

Algunos autores señalan que la función renal se altcra tardítmente por lo cual la hipertensión también es tardía y excepcional, desapareciendo postnefrectomía.

En tados los enfermos se encontró una masa abdominal palpable, con características tumorales. En 52,3\% fue del lado izquierdo y en 4 casos sobrepasó la linea media. En un $13 \%$ fue bilateral.

El estudio radiológico comprendió radiografía de tórax, radiografía abdomen simple y pielografía de eliminación. En 9 pacientes encontramos imágenes nodulates metastásicas en ambos campos pulmonares.

En 12 enfermos la pielografía mostró dilatación y alargamiento de cálices por masa intrarenal y en 8 se encontró exclusión renal, (Tabla 1).

Tabla 1

ESTUDIO RADIOLOGICO

\begin{tabular}{lcc}
\hline Tipo de examen & No Casos Positions & $\%$ \\
\hline Radiograf́a tórax & 9 & 25 \\
\hline Pielografía eliminac. & 20 & \\
- Distorsión cálices & 12 & 86 \\
- Exclusión renal & 8 & \\
\hline Radiogr. Abdomen & & \\
simple & 23 & 100 \\
\hline
\end{tabular}

En todos los casos la radiografía abdomen simple mostró una masa de tamaño variable que rechaza asas intestinales.

La interuención quirúrgica se indicó con carácter de urgente en todos los casos.

La via de acceso quinúrgico fue la transperitoneal (o anterior) que permite una mejor visualización del pedículo renal para ligar vasos rápidamente, especialmente la vena re- nal y cvitando con ello el desplazamiento dc células neoplásicas por esta vía, examinar el riñón opuesto, y tener una amplia visualiza. ción de toda la cavidad abdominal en busca de metástasis, (22). En 20 casos fue posible Ja extirpación total del riñón tumoral (Tabla 2), 3 enfermos fueron declarados inoperables por la gran extensión de la lesión con invasión de vasos importantes (vena cava) y de otros órganos (suprarrenat, higado, etc.).

Tabla ?

TRATAM: HALLAZGOS OPERATORIOS

Tumor extirpable totalmente -20 casos $(87 \%)$

a) $\operatorname{Sin}$ metŕstasis intraop.

- 10 casos

b) Con metástasis intraop.

- 10 casos

Tumor no extipable (invasor) - 3 casos (19\%)

Total casos

23 casos

En 10 pacientes se encontraron metástasis nacroscópicas en el acto quirúrgico: del higado en 4 casos y pulmones en 6 casos. Según diversos autores predomina la neoimplantación celular tumoral en pulmones, $(2,5,26)$.

La mortalidad intraoperatoria fue de 8 enfermos; en 2 de ellos existian metástasis pul. monares extensas bilaterales.

Fl grado de inzasion tumoral o etapa se determinó en base al protocolo operatorio y al examen histopatológico. En 16 de nuestros 23 pacientes encontramos compromiso vascular o capsulovascular (Tabla 3) que determinó la existencia de metástasis sistémicas, detectadas ya al ingreso en 8 enfermos, o aparecidas en el curso de la evolución postoperatoria en otros 8 enfermos.

Tabla 3

GRADO DE INVASION TUMORAL Y METASTASIS

\begin{tabular}{lrrr}
\hline & No Enf. Metdstasis & $\begin{array}{c}\text { Sistémicas } \\
\text { ingreso }\end{array}$ & postop. \\
\hline Invasión capsular & 3 & - & - \\
Invasión vascular & 3 & 4 & $\mathbf{4}$ \\
Invasión capsulovascular & 8 & 4 & 4 \\
Sin invasion & 4 & - & - \\
Total enfermos & $\mathbf{2 3}$ & $\mathbf{8}$ & $\mathbf{8}$ \\
\hline
\end{tabular}


a) Radioterapia: Se usó en $\mathbf{1 6}$ enfermos en dosis variables entre 3.000 y 5.000 rads. de cobalto por tiempo variable. Solamente un paciente recibio cobaltoterapia preoperatoria asociada a quimioterapia; 15 enfermos se tra. taron en el postoperatorio y 5 de ellos recibieron solamente radioterapia postoperatoria.

Tabla 4

\section{TRATAMHENTO EFEGTUADO}

\begin{tabular}{lcc}
\hline Tipo de tratamiento & No Enf. & Porcentaje \\
\hline Nefrectomia sola & 4 & 17,3 \\
Nefrect./Radiot. postop. & 5 & 21,7 \\
Nefrect./Radiot. Quimiot. & & \\
postop. & 9 & $39, \mathrm{I}$ \\
Nefrect./Radiot. Quimiot. & & \\
postop. & 1 & 4,9 \\
Nefrect./Quitriot. postop. & 1 & 4,3 \\
Ningún tratamiento (inop.) & 3 & 13,0 \\
Total enfermos & 23 & 100,0 \\
\hline
\end{tabular}

b) Quimioterapia: Se usó en el enfermo en periodo preoperatorio asociada a radioterapia y en 10 pacientes en el postoperatorio, también asociada a cobaltoterapia. Las drogas de elección fueron D-Act y VGR fundamentalmente, (en 2 niños se agregó Mtx y en uno, Endoxan) en dosis adecuadas a cada enfermo y por tiempo variable. Como único tratamiento se usó en un enfermo declarado inoperable, para disminuir la extensión de las metástasis pulmonares, (el niño falleció 6 meses después de la operación en su domi. cilio).

La nortalidad global de nuestra serie es de un $69,4 \%$ (16 pacientes), cifra que excede en mucho a la mortalidad señalada por autores extranjeros y da un sombrio pronóstico a nuestros niños portadores del tumor de Wilms.

La sobrevida es pobre: 7 enfermos $(30,4 \%)$ que son controlados cada 1-2 meses con exa. men clínico acucioso, radiografía de tórax, hemograma y VSH.
Del análisis de Ios resultados obtenidos creemos que hay algunos factores fundamentales que inciden en el pronóstico de estos enfermos.

a) Edad en el momento del diagnósico: Antes de la era de la quimioterapia el resultado del tratamiento del tumor de Wilms en el niño estaba en relación con la edad del enfermo en el momento del diagnóstico, con un mejor pronóstico en los menores de 2 años, (40). Según Collins, existe un perfodo de riesgo de recurrencia y un periodo de curación (9).

Pero, otros autores $(19,27)$ dicen que bastan dos años de sobrevida libres de recurrencia para considerar curado al enfermo.

En nuestra serie tenemos 2 pacientes con peligro de recurrencia y 5 estarian curados definitivamente Tabla 5 .

Relacionamos edad de comienzo y sobrevida post-tratamiento, y encontrarnos que los menores de 2 años alcanzan una mayor sobrevida y tienen, entonces, un mejor pronóstico (Gráfico 5) .

Tabla 5

SOBREVIDA

\begin{tabular}{ll}
\hline $\begin{array}{c}\text { Sobrevida global } \\
\text { Duración del control en } \\
\text { enfermos vivos }\end{array}$ & \\
\hline & \\
$0-6$ meses & 0 \\
$6-12$ meses & 1 \\
1-2 años & 1 \\
$2-4$ arios & 1 \\
$4-8$ affos & 2 \\
Curado definjitivamente & 5 enfermos $(22 \%)$ \\
Riesgo de recurrencia & 2 enfermos $(8,6 \%)$ \\
\hline
\end{tabular}

b) Estadio Clínico: Según el esquema de clasificación propuesto por Fleming (9) en 1970, ampliado por Pochedly (26, 27) en 1973, de nuestros casos encontramos que 10 de ellos corresponden al Grado IV y 3 al Grado Ji, estadios muy avanzados y prácticamente terminales, Tabla 6. 


\begin{tabular}{lcc}
\hline Estadio & No Casos & Porcentaje \\
\hline I & 4 & $17,4 \%$ \\
Ita & 2 & $8,7 \%$ \\
Irb & 2 & $8,7 \%$ \\
In2 & 2 & $8,7 \%$ \\
Inc & 3 & $13,0 \%$ \\
Iv & 10 & $43,5 \%$ \\
Total & 23 & $100,0 \%$ \\
\hline
\end{tabular}

En cambia, en el Grado t solo reunimos 4 casos.

Si relacionamos estos diferentes estadios con la sobrevida de los pacientes, vemos que los estudios I y $\mathrm{II}^{z}$ presentan la mayor sobrevida, (Tabla 7).

En el estadio $111^{2}$ hay un $50 \%$ de sobrevida, que no to consideramos valedero de ningún modo ya que el número de casos es insuficiente

\section{SOBREVIDA}

\begin{tabular}{lccc}
\hline Fstadio & N9 Enf. & Enf. vivos & $\%$ Sobrevida \\
\hline I & 4 & 4 & $100 \%$ \\
Ila & 2 & 2 & $100 \%$ \\
Inb & 2 & 0 & $0 \%$ \\
IIIa & 2 & 1 & $50 \%$ \\
Inc & 3 & 0 & $0 \%$ \\
IV & 10 & 0 & $0 \%$ \\
Total & 23 & 7 & $30 \%$ \\
\hline
\end{tabular}

para tener significado estadístico, y el tiempo de control es muy corto como para determinar curación definitiva. El estadio iv no presenta sobrevidas.

De acuerdo con el criterio de Mahesh Kumar y cols. (20) para juzgar el graclo de inva. sión tumoral y de acuerdo al informe histopatológico, (Tabla 8) vemos que existiendo invasión vascular o capsulovascular, la sobrevida es nula.

TabJa 8

RELACION ENTRE GRADO DE INVASION

TUMORAL Y SOBREVIDA

\begin{tabular}{|c|c|c|c|c|c|}
\hline No enf. & \multicolumn{2}{|c|}{ Hallazgo histopatolog. Curacion } & \multicolumn{2}{|c|}{ Riesgo recurr, Fall. } & \multirow{2}{*}{$\begin{array}{l}\text { Sobrev. } \\
100 \%\end{array}$} \\
\hline 3 & Invesión capsular & I $(n a)$ & 2 (IL) & - & \\
\hline 8 & Invasión vascular & - & (xia) & 8 & $0 \%$ \\
\hline a & Invasión caps. vasc. & $\rightarrow$ & $\longrightarrow$ & 8 & $0 \%$ \\
\hline \multirow[t]{2}{*}{4} & Sin invasión & $4(\mathrm{t})$ & - & 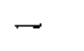 & $100 \%$ \\
\hline & & & - & & \\
\hline
\end{tabular}

Tratamiento efectuado

Según la literatura, los mejores resultados se obtienen ofreciéndole al enfermo cirugía radical precoz y asociada a quimioterapia y/o $\overrightarrow{r a}$ dioterapia, adaptada a cada estadio clínico, $(8,13,27,29)$. Estas experiencias dan cifras de sobrevida que fluctúan entre 36 y $89 \%,(20)$.
En nuestros pacientes, encontramos que la nefrectomla ofrecida como único tratamiento no sirve en las etapas avanzadas del tumor ya que los pocientes fallecen todos, más tarde o más temprano, por metástasis; (no tuvimos ningún caso de recurrencia neoplástica focal), (Tabla 9). Influye aquí lo avanzato de la lesión tumoral. 


\begin{tabular}{|c|c|c|c|c|}
\hline & \multirow[t]{2}{*}{ No Enf. } & \multirow{2}{*}{$\begin{array}{l}\text { Estadio } \\
\text { Clinico }\end{array}$} & \multicolumn{2}{|c|}{ Sobrevida } \\
\hline & & & No Enf. & $\%$ \\
\hline Netrectomfa pura & 4 & $\mathrm{sv}=1$ & 0 & 0 \\
\hline Nefrect./Radiot. Postop. & 5 & $\begin{array}{r}\mathrm{r}=1 \\
\mathrm{Ia}=1 \\
\mathrm{IIc}=1\end{array}$ & 2 (c) & 40 \\
\hline & & $\begin{aligned} I V & =2 \\
I & =3\end{aligned}$ & $5(8 \mathrm{c})$ & 55 \\
\hline $\begin{array}{c}\text { Nefrect, } / \text { Radiot.-Quimiot } \\
\text { Postop. }\end{array}$ & 9 & $\mathrm{JI}=1$ & $\begin{array}{l}(2 \mathrm{r}) \\
0\end{array}$ & 0 \\
\hline Nefrect. /Ractiot.-Quimiot. & & $\begin{aligned} \mathrm{IIb} & =2 \\
\mathrm{Itt} & =2\end{aligned}$ & 0 & 0 \\
\hline Pre y Postop. & l & $I V=1$ & 0 & 0 \\
\hline Nefrect./Quimiot. Postop & 1 & $1 v=1$ & 7 & \\
\hline Ninguin tratarniento (Inop) & 8 & nuc $=1$ & & \\
\hline Total Enfermos & 23 & Inc $=1$ & & \\
\hline
\end{tabular}

Combinando cirugía con radioterapia y quimioterapia postoperatoria en dosis adecuadas y por tiempo suficiente, se logró una mayor sobrcvida de los pacientes, $(55 \%)$.

Solamente encontramos un paciente trata. do con cirugía y quimioterapia postoperatoria, en el estadio inc: fallece durante una intervención motivada por oclusión intestinal por briclas. Comesponde a uno de los tres casos bilaterales. Cinco enfermos recibieron radioterapia postoperatoria: 2 de ellos están definitivamente curados estadios $x$ y na $y$ fallecieron.

La gran mayoría de nuestros enfermos tc. nia invasión vascular o capsulovascular, lo que significó fracaso del tratamiento efectuado en el $100 \%$ de estos casos. Sin invasión tumo. ral encontramos 4 nifíos; todos ellos recibieron radiotetapia y quimioterapia postnefrectomía evolucionando satisfactoriamente. Después de dos años de control sin recurrencia focal y sin signos de metástasis, los consideramos curados definitivamente.

\section{CONCLUSIONES}

A pesar de que los métodos de tratamiento han progresado en los últimos años, y de que el control de estos enfermos se está haciendo por un Equipo Oncológioo múltiple, el pro. nóstico del tumor de Wilms sigue siendo reservado, en nuestro medio.

Lo mayoría de nuestros pacientes inició su tratamiento en estadios evolutivos avanzados, lo cual concliciona mala respuesta y elevada mortalidad. Es imperativo efectuar un diagnóstico lo más precoz posible para aspirar a una mejor sobrevida. Para ello debemos tener presente dos conocidas sentencias: "Toda masa palpable abdominal $\epsilon n$ un niño pequeño debe considerarse maligna, hasta que se demuestre lo contrario".

"Todo tumor abdominal constituye una urgencia relativa, que debe tener diagnóstico y conducta terapéutica esclarecida dentra del más breve plazo de tiempo".

\section{RESUMEN}

Se presentan 23 casos de Tumor de Wilms del Hospital Roberto del Río entre 1962-1974. Todos fueron explorados quirúrgicamente. En 3 no fue posible la extirpación, solo se tomó biopsia. La mayor parte de ellos recibió Radioterapia y/o Quimioterapia postoperatoria.

La sobrevida lograda en este período es baja, $30 \%$, condicionada en parte por lo avanzado de las lesiones en el momento de la ope- 
ración. El $70 \%$ de estos pacientes tenian ya invasión vascular a cápsulo vascular.

\section{SUMMARY}

23 cases of Wiln's Tumor seen at Hospital Roberto deI Rio in Santiago de Chile, from 1962-to 1974 are reviewed. The cure rate in only $30 \% .70 \%$ of this serie had vascular and capsulo-vascular invation at operation.

\section{REFERENCIAS}

1. Anderson, E. ct als.: "Bilateral Wilm's Tumor: Diagnogis and management" Clin. Ped. 7: 596.99.

2. Beckwith, J. R.: Mesenchywal tenal neoplans of Infancy. J. Ped. Surg. 5: 405-406, 1970.

3. Bjelke, E.: Malignant necplams of the kidncy in children. Cancer 17: \$18-21, 1964.

4. Beresi, $v$ y cols.: Tumor de Wilms en la infancfa Rev. Chil. Ped, 44: 65-72, 197 9.

5. Dargeon, H.: Cancer in Childhood. St. Louis C. V. Mosby Co., 1940.

6. Emparanza, E.: Tumores maligncs en la infancia. Rer. Chil. Ped. 39: 307-13, 1968.

7. Favar, B. E.: Renal tusnors in the nconatal period. Cancer 22: 845-55, 1968.

8. Fernbach, D. J. et als.: Role ofactinomycin in the inproved survical of children with Wilen's Tumor. J. A. M. A. 195: 1005-9, 1966.

9. Fleming, I. et als.: Glinical and patbologic stating as a guide in the managetrent of Wilm's Tumor. Cancer 26: 660-665, 1970 .

10. Fraumen, J. F. et als.: Wilm's tumor and congenital hemihipertrophy: report of five new cases and review of literature. Pcdi 40: 886, 1967.

11. Gross, R. E.: Enbryoma of the kidney (Wilm's numor). The surgery of infance and Childhood. Chap. 45; W. B. Sanders Co. Philadelplia and London, 1953 .

12. Howatr, J. y cols: Tumores en la infancia. Rev. Chil. Ped. 40: 522-37, 1969.

15. Howard, R. et als. Actinomycin $D$. in Wilm's tumor: treatment of Iung metastases. Arch. Dis. Childhood 40: 200.02, 1965.
14. Jagasia, $\mathrm{K}, \mathrm{H}$. et als.: Congenital anomalics of the kidney in association with Wilna's tumor. Ped. 35: 398-40, 1965.

15. Jdung, R. B.: Urinary vanil-mandelic acid f'xcretion in clitdren. J. Ped. 62: 841.54, 1963.

16. Kuffer, F, : Surgical complications in clitilden unrlergoing cancer therapy. Am. Surg. 167: 215-10, 1968.

I7. Iattimer, J. K.: The place of surgery in Wiln's Tumors. J. A. M. A. 204: 985-86, 1968.

18. Lattimer, J. K. et als.: Prognosis more favorable in infants under one year of age. J. A. M. A. 1 fl: $2163-68,1959$.

19. Jaurende, B. et als.: WViln's tumor prior to onset of hemihipertrophy. Am. J. Dis Children, 120: 565-65, 1970.

20. Lelong, M.: Aclualités Pediatriques, Edit. Boim Y Cia. 1961.

21. Nahesh Kumar, A. P.: Capsular and vascular inva. sion: Important prognostic factors in Wilm's tumor. J. Ped. Surg. 10: 301-309, 1975.

22. Malaneat, A.: Diagnóstico de quiste renal y carci. noña del rif̂́on. Clin. Quirúrg. N. A., 1377.1391, 1965.

23. Martin, L. W.: Bilateral nephroblastona (Wilm's cumor) of the kidney. Ired. 28: 101, 1961.

24. Martin, L. W. et als.: An evaluation of tetn years expexience with retroperitoneal limph node disection for Wilm's tumor. J. Ped. Surg. 4: 683-87, 1969.

2.. Nelson, W.: Tratado de Pediatrfa. Edit. Salvat S. A Barcelona, $197 \mathrm{~L}$.

26. Neuhauser, En: Wilt's Tumor: Rarliology - Pow. graduate Melicine 47: 55, 1970.

27. Olmedo, R.: Tumor de Wilms. Actualidades on Cancerologia. Soc. Chil. Cancerologia, 1965.

28. Pochedly, C.: Wilm's tumor I: Clinical features and tumor biology. New York. J. Med, 71: 1089.93, 1971.

29. Pochedly, C.: Wilm's tumor II: Diagnosis and Treatment. New York. J. Med. 71; 1205-209, 1971.

30. Pochedly, C.: Mayor problems in Clilldhood Canocr. Charles C. Thomas Publisher, Springfield, Illinois, U.S.A. 1971. 
31. Pratt, Cit, B.: The management of tutnots solids in clitdren. Clin. Pad. N. A.

92. Richmonil, H. Neonatal renal Lumons. J. Ped. Surg. b: 413-18, 1970 .

33. Samuels, L. D.: Organ scan diagrosis of abdominal masses in children. $J$. Ped. Surg. 6: 124-91, 1971 .

34. Smyder, H. E: Bilatcral Wilan's tumor. Am. J. Surg. 110: 492-96, 196.5.

35. Sutow, W. et als.: Comparisson of survical curvs 1956 versus 1962 in children with Wilm's tumor and neuroblastonia. Perf. 45: 800-11, 1970.

96. Sutow, W.: Clinical Pediatric Oncology. The C. V. Mosby Company St. Louis 359-80. 1973.

37. Taricoo, s. y cols.: Tumos de Wilms. Rev. Chil, Ped. 9: 908, 1970.
\$8. I'rrizola $y$ cols.: Tumores abdominales en la infancia. Rev. Clis. Ped. 40: 898-905, 1969.

39. Wagget, J.: Wilm's tumor: precperativeradiotherapy and clicmotherapy in the management of massivc tumors. Cancer, 26: $338-40,1970$.

40. Waisman, J. B.: Renal neoplasus of thenewborn. J. Pcrl. Surg. 5: 407-12, 1970.

41. Williams, J. G.: Tumores en el niño. Edit. Jims, Barcelona, 1975 .

42. Wolff, J. $A$. et als.: "Single versus multiple course Actinoniycin-Therapy of Wilm's tumor". The $N$. Engcl. J. Merdicine, 290: 84-86, 1974.

43. Wolff, J. A. et als: "advances in the treatment of Wilm's tumor". Cancer, 35: 90I-904, 1975.

44. Mess Redonda: Tumores en la infancia, Rev, Chil. Pcd. 7: 522, 1969. 